\title{
LDL Lowering Drugs: Current Perspectives
}

\section{Norata Giuseppe Danilo1,2,3*}

${ }^{1}$ Department of Pharmacological Sciences, Università degli Studi di Milano, Italy

${ }^{2}$ Center for the Study of Atherosclerosis, Società Italiana Studio Aterosclerosi, Ospedale Bassini, Cinisello Balsamo, Italy

${ }^{3}$ The Blizard Institute, Centre for Diabetes, Barts and The London School of Medicine and Dentistry, Queen Mary University, London, UK

The discovery of statins has provided a very effective approach in reducing dyslipidemia and cardiovascular risk as documented by the results obtained in clinical trials and in clinical practice and lead to the "motto" for LDL lowering approaches "the lower.... the better". Despite this, in high-risk and very high risk patients, the current efficacy of statins comes short of providing the benefit that could derive from a optimal reduction of LDL cholesterol (LDL-C) and, as the elevated doses of statins required in these patients increase the risk of side effects, many patients do not achieve at the maximal tolerated dose the recommended goals for LDL-C, non-HDL-C, and apoB. This clearly prompted in the last decade, the research towards the identification of new targets by re-evaluating key enzymes involved in lipid and lipoprotein metabolism and by taking advantage from the genetic insights deriving from large genome wide association studies. Some of these findings were quickly traduced in therapeutic options and as of the end of 2012 two molecules controlling liver lipoprotein synthesis, namely mipomersen and lomitapide were approved by the FDA and the EMA while antibodies blocking the activity of proprotein convertase subtilisin/kexin type 9 (PCSK9) and resulting in increased LDL-R efficiency entered phase III of clinical development.

In details, mipomersen is an anti sense oligonucleotide (ASO) targeting apoB and leading to a dose-dependent reduction in apoB and total cholesterol. Following hybridization to the mRNA, the ASO inhibits translation and splicing, and leads to degradation of the apoB mRNA by RNase. Overall, mipomersen provided significant further reduction in LDL-C ( $\sim 30 \%$; range -21 to $-37 \%)$ and other lipids when added to conventional lipid lowering therapy. The most common adverse effects were injection-site reactions and flu-like symptoms. Liver fat accumulation was also observed in both phase II and phase III studies and is in line with the mechanism of action of the drug [1].

Additional studies are needed to confirm the lipid effects and to provide more information on incidence of adverse effects, especially on fat accumulation and potential inflammatory response(s) in the liver, as well as compliance. Lomitapide inhibits the microsomal triglyceride transfer protein (MTP) which is a key protein found in the endoplasmic reticulum of hepatocytes and enterocytes, that mediates the formation of apoB-containing lipoproteins in the liver and in the intestine. The drug, tested in monotherapy in patients with homozygous Familial Hypercholesterolemia (FH) showed a reduction in circulating LDL-C, apoB, total cholesterol, non-high-density lipoprotein cholesterol (nonHDL-C) and Lp(a) levels [1,2]. The most common adverse events were gastrointestinal symptoms. Elevations in liver transaminases (5-11 times ULN) occurred in 4 patients and resolved with dose reduction or temporary suspension of the study drug. No patients discontinued treatment because of liver function abnormalities.

Currently, the two drugs are approved for the treatment of patients with homozygous familial hypercholesterolemia; ongoing studies of mipomersen and lomitapide should provide additional information on the safety and tolerability in terms of liver steatosis and potential patient populations for whom they may be appropriate. PCSK9 is a serine protease which acts both intracellular, as a chaperone directing the LDL-R to the lysosomes, and in the circulation, by promoting LDL-R internalization, these processes clearly promotes the degradation of LDL-R $[3,4]$. The possibility of inhibiting PCSK9 represents therefore a logical step to enhance the lipid-lowering effect of conventional agents such as statins which work through the increase of LDL-R expression on the hepatocyte surface, followed by increased LDL turnover and reduction of plasma cholesterol levels. To data, at least five different human monoclonal antibodies and three gene-silencing approaches are under development. Among a series of antibodies against PCSK9, clinical trial results are available for two of them, SAR236553/ REGN727 and AMG145 and these compounds are both in phase II or III development. The safety results for PCSK9 monoclonal antibodies are encouraging however further trials are therefore required to test the long-term safety and efficacy of PCSK9 monoclonal antibodies in larger and more varied patient populations.

In conclusion, several new therapeutical options for the management of dyslipidemia are available or will become available soon. While the use is so far restricted to high risk patients with genetic hypercholesterolemia, data from the ongoing clinical studies will help to clarify whether a wider number of patients with dyslipidemia could be benefited from these therapies.

\section{References}

1. Norata GD, Ballantyne CM, Catapano AL (2012) New therapeutic principles in dyslipidaemia: focus on LDL and $L p(a)$ lowering drugs. Eur Heart $J$ in press.

2. Cuchel M, Meagher EA, du Toit Theron H, Blom DJ, Marais AD, et al. (2012) Efficacy and safety of a microsomal triglyceride transfer protein inhibitor in patients with homozygous familial hypercholesterolaemia: a single-arm, openlabel, phase 3 study. Lancet.

3. Tibolla G, Norata GD, Artali R, Meneghetti F, Catapano AL (2011) Proprotein convertase subtilisin/kexin type 9 (PCSK9): from structure-function relation to therapeutic inhibition. Nutr Metab Cardiovasc Dis 21: 835-843.

4. Norata GD, Garlaschelli K, Grigore L, Raselli S, Tramontana S, et al. (2010) Effects of PCSK9 variants on common carotid artery intima media thickness and relation to ApoE alleles. Atherosclerosis 208: 177-182.

*Corresponding author: Giuseppe Danilo Norata, Department of Pharmacologica and Biomolecular Sciences, University of Milan, Milan, Italy, Tel: +39 0250318313 . Fax: +39 02 50318386; E-mail: Danilo.Norata@unimi.it

Received November 20, 2012; Accepted November 21, 2012; Published November 23, 2012

Citation: Danilo NG (2013) LDL Lowering Drugs: Current Perspectives. Cardiol Pharmacol 2:e104. doi:10.4172/2329-6607.1000e104

Copyright: (c) 2013 Danilo NG. This is an open-access article distributed under the terms of the Creative Commons Attribution License, which permits unrestricted use, distribution, and reproduction in any medium, provided the original author and source are credited. 